J. Austral. Math. Soc. 21 (Series A) (1976), 473-480.

\title{
THE CHROMATIC NUMBER OF THE SPHERE
}

\author{
Dedicated to George Szekeres on his 65th birthday \\ GUSTAVUS J. SIMMONS \\ (Received 24 January 1975) \\ Communicated by Jennifer Seberry Wallis
}

\begin{abstract}
Erdös, Harary and Tutte have defined the chromatic number of the plane to be the least number of sets partitioning the plane such that no set contains two points at unit distance apart. By analogy, the chromatic number $\chi\left(S_{r}\right)$, of the sphere, $S_{r}$ of radius $r$ is defined to be the least number of sets partitioning the surface of $S$, such that no set contains two points at unit chordal distance apart. In this paper it is proven that

$$
4 \leqq \chi\left(S_{r}\right) \quad \text { for } \quad 1 / \sqrt{3} \leqq r
$$

and that this bound is best possible since

$$
4=\chi\left(S_{1 / \sqrt{ }}\right) .
$$

Define the chromatic number, $\chi\left(S_{r}\right)$, of the sphere, $S_{r}$, of radius $r$ to be the least number of sets partitioning the surface of $S_{r}$ such that no set contains two points at unit chordal distance apart. The general question of determining the value of $\chi\left(S_{r}\right)$ appears to be new to the literature, however, values for at least two special cases have been conjectured by P. Erdös. For the plane, $S_{\infty}$, Erdös, Harary and Tutte (1965) introduced the terminology chromatic number of the plane for $\chi\left(S_{x}\right)$. In this case it is well known that $4 \leqq \chi\left(S_{x}\right) \leqq 7$; Hadwiger, Debrunner and Klee (1964), Moser and Moser (1961), Woodall (1973). One of Erdös' long standing conjectures is that $\chi\left(S_{x}\right)=4$. More recently Erdös had conjectured that it was possible to 3-color the unit sphere so that every inscribed equilateral triangle of side $\sqrt{3}$ would have one vertex of each color, i.e. that $\chi\left(S_{1 / \sqrt{ } 3}\right)=3$. Simmons (1974) disproved this by showing $4 \leqq \chi\left(S_{1 / \sqrt{ } 3}\right)$ while Straus
\end{abstract}

This work was supported by the United States Energy Research and Development Administration. 
established an upper bound of 5 by constructing a permissable 5-coloring. In this paper we show that $4 \leqq \chi\left(S_{r}\right)$ for all $1 / \sqrt{3} \leqq r$ using a generalization of the argument used for $\chi\left(S_{1 / V^{3}}\right)$. It is interesting that the case $r=1 / \sqrt{3}$ chosen by Erdös for one of his conjectures is one of the two degenerate cases (in a sense to be explained shortly) which must be disposed of by special arguments.

The key element in the constructions which follow is an equilateral diamond consisting of two unit side equilateral triangles sharing a common base edge.

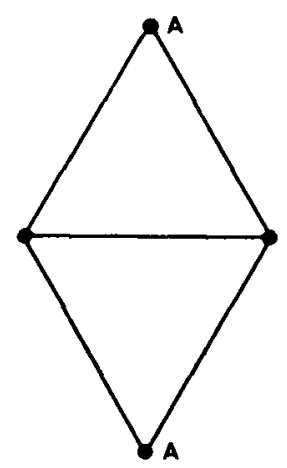

For all $1 / \sqrt{3} \leqq r$ it is obviously possible, by folding the diamond about the common base edge, to inscribe it in $S_{r}$ so that all four vertices lie on the surface of the sphere. Figure 1 illustrates this for $S_{0.58}$. The important property of the inscribed equilateral diamond to this paper is that in any 3-coloring of $S_{r}$ the two tip vertices, $a$, must be colored alike. Hence if the diamond is rotated about the diameter through one tip vertex, the circle swept out by the other tip vertex must be colored like the fixed vertex.

In Figure 1, two parameters are labeled; $l$ and $x$. The chordal distance, $l$, between the tip vertices of the inscribed diamond is given in terms of the radius of $S_{r}$ by

$$
l=2 \sqrt{\frac{3 r^{2}-1}{4 r^{2}-1}}
$$

which clearly achieves a maximum value of $\sqrt{3}$ at $r=\infty$, i.e. the height of the equilateral diamond when drawn in the plane. The minimum distance from one tip vertex of the diamond to the diameter through the other tip vertex, $x$, is given by either

$$
x=\left|\frac{2\left(2 r^{2}-1\right)}{r\left(4 r^{2}-1\right)} \sqrt{3 r^{2}-1}\right|
$$


or

$$
x=\left|\frac{l\left(l^{2}-2\right)}{\sqrt{4-l^{2}}}\right| .
$$

Define $\alpha$ to be the acute central angle between the radius to the rotating tip vertex and the diameter through the fixed tip vertex, then

$$
\sin \alpha=\frac{x}{r}
$$

As has already been noted, if a 3-coloring of $S$, is possible then when the inscribed diamond is rotated about the diameter through one tip vertex, the other tip vertex must describe a monochrome circle of radius $x$. If $\frac{1}{2} \leqq x$, then at least four colors are obviously needed to color $S_{r}$ since a chord of length 1 could be placed in this monochrome circle - to contradict the assumption that $S_{\mathrm{r}}$ had

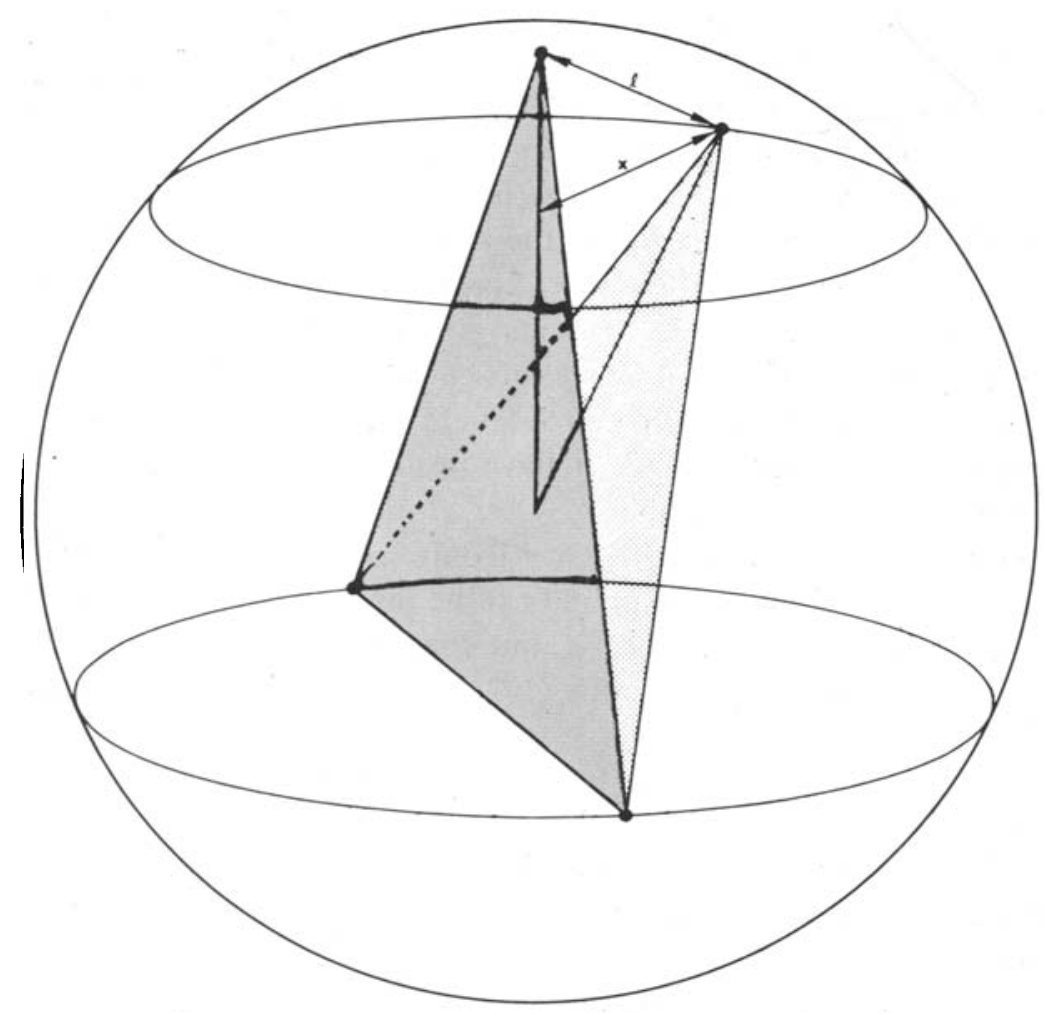

Figure 1 


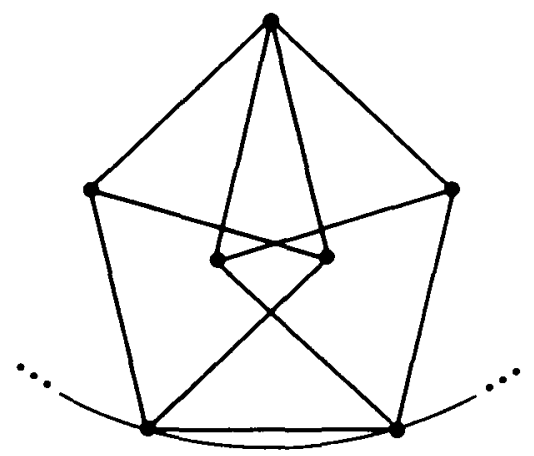

Figure 2

been properly 3-colored. Incidentally, this construction in the plane gives the finite subgraph used by the Mosers' (1961) to prove that $4 \leqq \chi\left(S_{x}\right)$.

If $0<x<\frac{1}{2}$, then every point on the monochrome circle swept out by the rotating tip vertex could be taken as the fixed tip vertex and the diamond rotated in turn about the diameter through that vertex to form a closed monochrome cap of half angle $2 \alpha$, where $\alpha$ is given by Equation (4). A similar operation is then again possible on the points at the edge of this cap to give a new monochrome cap of half angle $3 \alpha$ etc., until finally the maximum chord length inscribable in the cap exceeds 1 . This again leads to a contradiction of the assumption that $S_{r}$ has been properly 3-colored. However if $x=0$, then the two tip vertices are both fixed under the rotation - and hence this construction does not contradict the assumption of a proper 3-coloring of $S_{r}$. There are, therefore, three cases to be considered: $0<x<\frac{1}{2}$ and $\frac{1}{2} \leqq x$ which have just been treated and $x=0$ which is considered in the next paragraph.

From Equation (2) one sees that $x=0$ only for $r=1 / \sqrt{3}$ and $r=1 / \sqrt{2}$. The only way that the equilateral diamond can be inscribed in $S_{1 / \sqrt{ } 3}$ is by folding it completely closed on itself to form a unit edge equilateral triangle. This is the special case dealt with by Simmons (1974) in his Magen David construction where it was shown that

$$
4 \leqq \chi\left(S_{1 / \vee 3}\right) \leqq 5 .
$$

The equilateral diamond, when inscribed in $S_{1 / v^{2}}$ has the two tip vertices diametrically opposed, i.e. the diamond becomes two faces of an inscribed octahedron.

To see that $\chi\left(S_{1 / \sqrt{ } 2}\right)=4$ in this case we first show that a 3 -coloring is impossible and then exhibit a suitable 4-coloring found by J. Davis. Assume three colors, say red, green and blue, suffice - then clearly; 1 . every great circle 


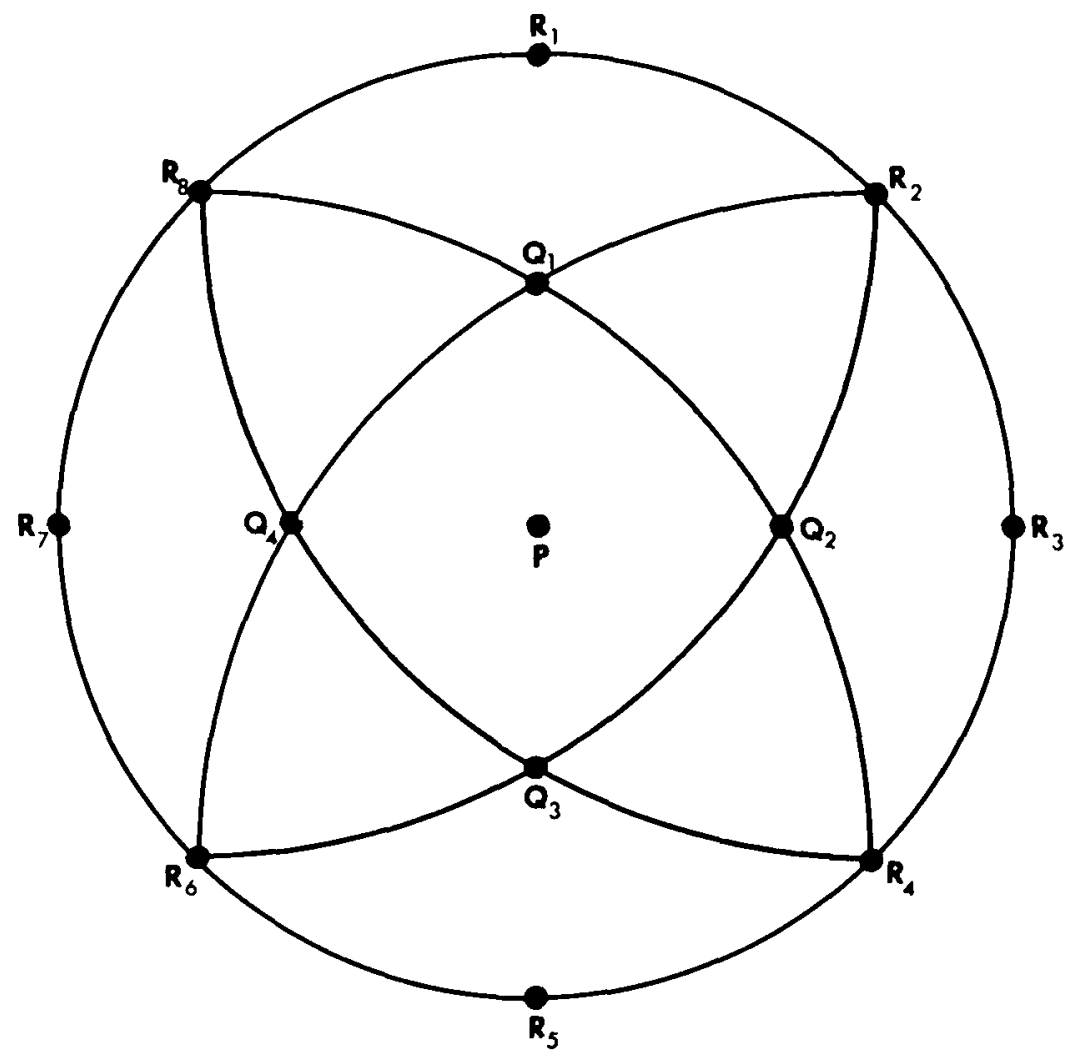

Figure 3

must be 2-colored, 2. every pair of diametrically opposed points must be the same color, and 3. any pair of points $90^{\circ}$ separated on a great circle must be colored differently.

Figure 3 shows a polar projection of $S_{1 / \sqrt{ } 2}$ in which $P$ is one tip vertex of the inscribed diamond, the outer circle through the points $R_{i}$ is the equator swept out by the rotating mid vertices, and arcs $R_{2} Q_{1} Q_{4} R_{6}, R_{2} Q_{2} Q_{3} R_{6}$, $R_{8} Q_{1} Q_{2} R_{4}$ and $R_{8} Q_{4} Q_{3} R_{4}$ are great circles symmetrically inclined with respect to the polar arcs so that $Q_{1}$ and $Q_{3}$ are $90^{\circ}$ apart on the great circle $R_{1} Q_{1} P Q_{3} R_{5}$ and $Q_{2}$ and $Q_{4}$ are $90^{\circ}$ apart on the great circle $R_{7} Q_{4} P Q_{2} R_{3}$. Let $P$ be red, then the equator is blue-green. Assume $R_{1}$ is blue, then $R_{5}$ is also blue and $R_{3}$ and $R_{7}$ are necessarily green. With no loss of generality let $R_{2}$ be blue, so that $R_{6}$ is also blue and $R_{4}$ and $R_{8}$ are green. Since the great circle through $R_{7} Q_{4} P Q_{2} R_{3}$ is red-green and $Q_{2}$ and $Q_{4}$ are $90^{\circ}$ apart either $Q_{2}$ is red and $Q_{4}$ green or vice versa. First, assume $Q_{4}$ is red and $Q_{2}$ is green then the great 

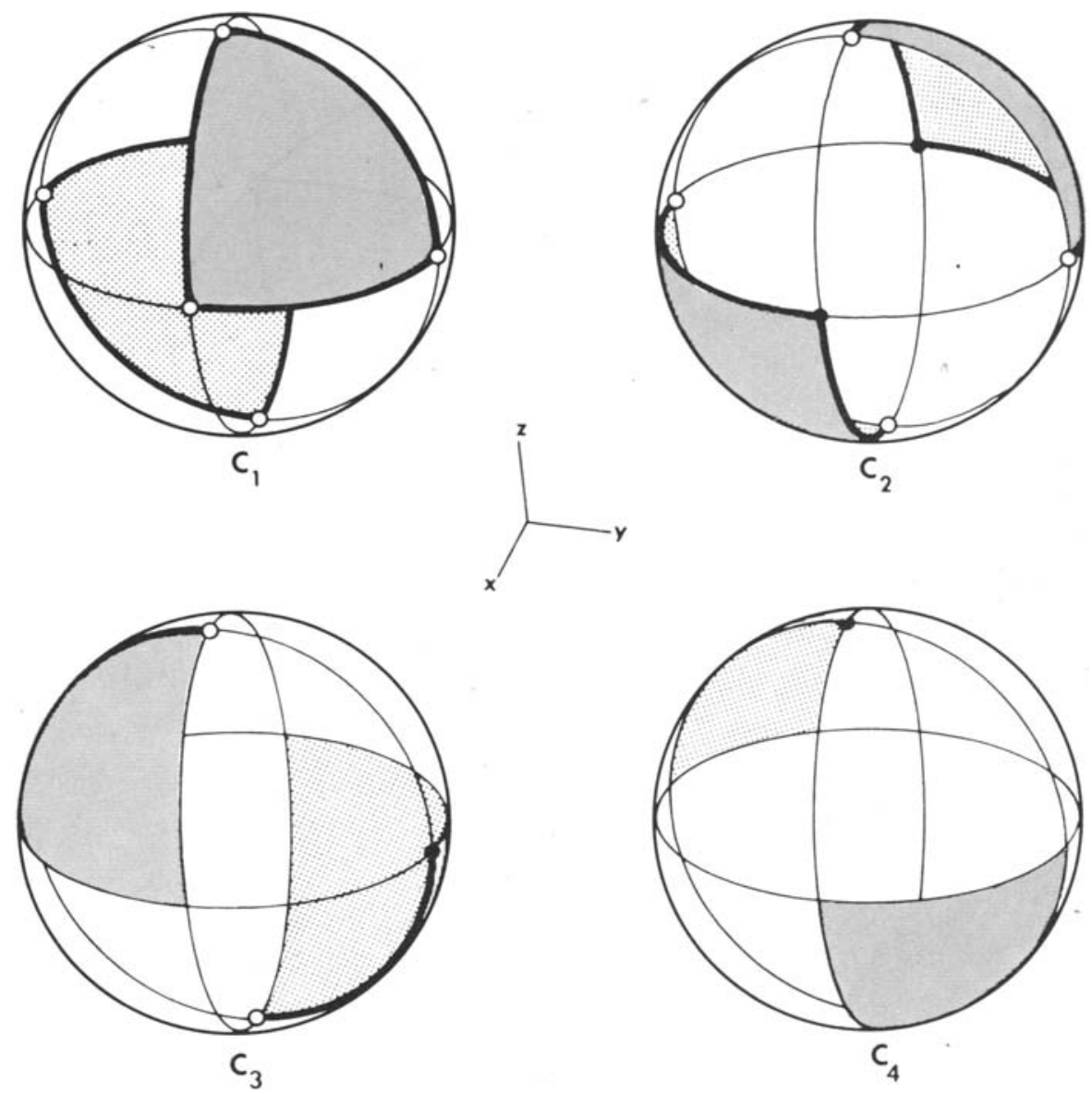

Figure 4

circle through $R_{8} Q_{4} Q_{3} R_{4}$ is red-green while the one through $R_{2} Q_{2} Q_{3} R_{6}$ must be blue-green. Thus $Q_{3}$ must be green - but this is impossible since it lies on the blue-red great circle through $R_{1} Q_{1} P Q_{3} R_{5}$. If $Q_{4}$ is green and $Q_{2}$ red, then the same argument shows that $Q_{1}$ must be green, etc. Hence a three coloring is impossible.

A permissable 4-coloring of $S_{1 / \sqrt{ } 2}$ is the following partition into four sets, $C_{i}$ of points $(x, y, z)$ where

$C_{1}-x, y$ and $z$ are of the same sign $\cup(a, b, 0) \cup(a, 0, b) \cup(0, a, b)$ where $a b>0$

$C_{2}-x$ is different in sign from $y$ and $z \cup(a, b, 0) \cup(a, 0, b) \cup( \pm 1,0,0)$ where $a b<0$ 
$C_{3}-y$ is different in sign from $x$ and $z \cup(0, a, b) \cup(0, \pm 1,0)$ where $a b<0$.

$C_{4}-z$ is different in sign from $x$ and $y \cup(0,0, \pm 1)$

Figure 4 illustrates this partition, where a bold boundary or solid vertex indicates that the points are to be included in the shaded set. It is of some interest to know where the transitions between the various cases occur. Figure 5 plots $r$ and $x$ as functions of $l^{2}$ which was chosen for the abscissa rather than $r$ since $0 \leqq l^{2} \leqq 3$, while $r$ is unbounded. The upper curve is $r$, since $x \leqq r$ in all cases. Equality holds only for the two indicated values

$$
l=r=\sqrt{\frac{7 \pm \sqrt{ } 17}{8}}
$$

$x=\frac{1}{2}$ at the values of $r$ corresponding to the three roots of Equation (5)

$$
4 u^{3}-16 u^{2}+17 u-4=0
$$

where

(6)

$$
r=\frac{1}{2} \sqrt{\frac{4-u}{3-u}}
$$

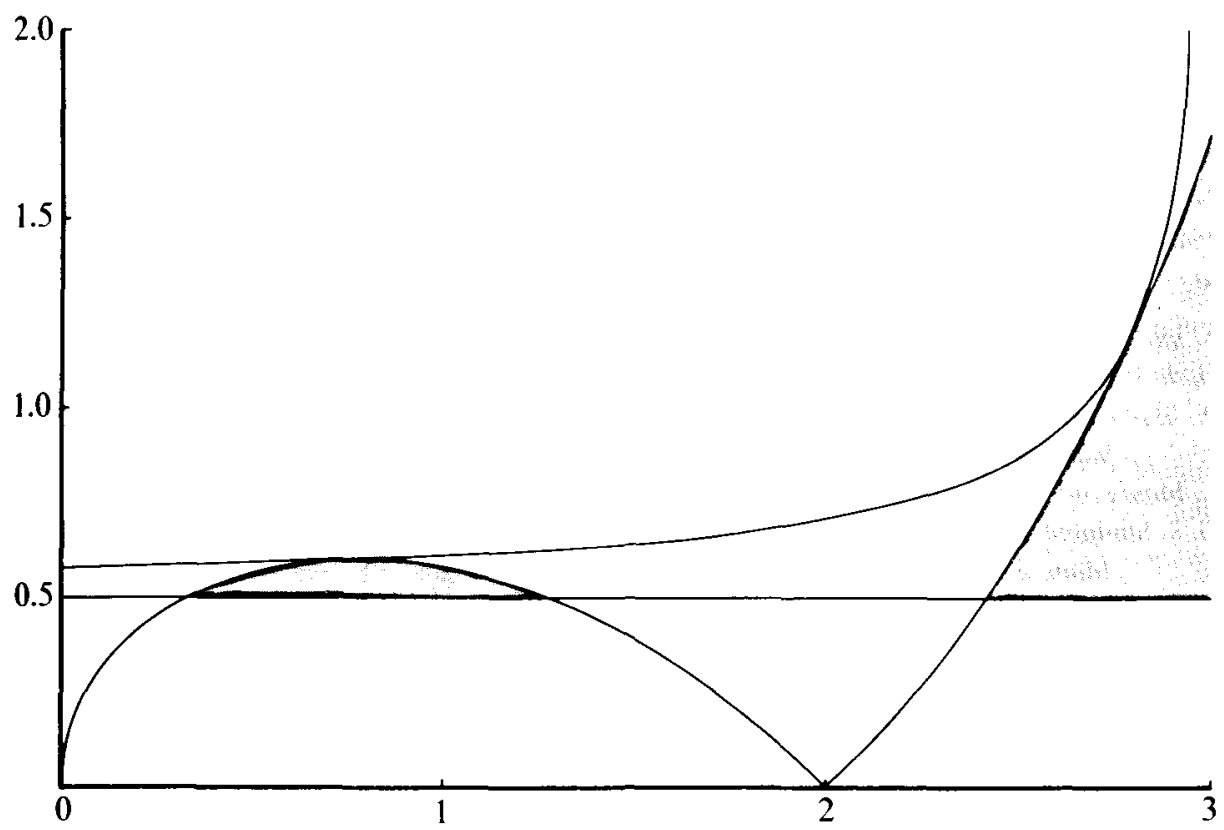

Figure 5 
Hence, we have $\frac{1}{2} \leqq x$, i.e. the second case, only for the approximate ranges.

$$
.586,158 \leqq r \leqq .627,745
$$

or

$$
.819,417 \leqq r
$$

which have been shaded in Figure 5.

The proofs by contradiction given earlier showing that $4 \leqq \chi\left(S_{r}\right)$ for $1 / \sqrt{3} \leqq r$ and $x \neq 0$ combined with the results for the two special cases where $x=0$ of $4 \leqq \chi\left(S_{1 / \sqrt{ } 3}\right) \leqq 5$ from Simmons (1974) and $4=\chi\left(S_{1 / \sqrt{ } 2}\right)$ given here prove that

$$
4 \leqq \chi\left(S_{r}\right) \quad \text { for } \quad 1 / \sqrt{3} \leqq r .
$$

The fact that $4=\chi\left(S_{1 / \sqrt{ } 2}\right)$ also shows that this is the best possible uniform bound.

Trivially $\chi\left(S_{r}\right)=1$ for $r<\frac{1}{2}$ and $\chi\left(S_{\frac{1}{2}}\right)=2$, so that the lower bound for $\chi\left(S_{r}\right)$ is only an open question for the range of radii $\frac{1}{2}<r<1 / \sqrt{3}$.

It seems appropriate to close with two new conjectures about the chromatic number of the sphere and a reiteration of Erdös' open conjecture on the chromatic number of the plane:

$$
\begin{array}{ll}
\text { 1. } & 4 \leqq \chi\left(S_{r}\right) \text { for } \frac{1}{2}<r \\
\text { 2. } & 4=\chi\left(S_{1 / \sqrt{3}}\right) \\
\text { 3. } & 4=\chi\left(S_{x}\right) .
\end{array}
$$

\section{References}

P. Erdös, F. Harary and W. T. Tutte (1965), 'On the dimension of a graph', Mathematika 12, 118-122.

H. Hadwiger, H. Debrunner and V. Klee (1964), Combinatorial Geometry in the Plane, Holt, Rinehart \& Winston, New York.

L. Moser and W. Moser (1961), 'Problem and solution P10', Canad. Math. Bull. 4, 187-189.

G. J. Simmons (1974), On a problem of Erdös concerning a 3-coloring of the unit sphere, Discrete Math. 8, 81-84.

D. R. Woodall, (1973), 'Distances realized by sets covering the plane', J. Combinatorial Theory Series A 14, 187-200.

Department of Mathematics

Sandia Laboratories

Albuquerque, New Mexico, 87115

U.S.A. 